\title{
Excitonic behavior in self-assembled InAs/GaAs quantum rings in high magnetic fields
}

\author{
N. A. J. M. Kleemans, ${ }^{1}$ J. H. Blokland, ${ }^{2}$ A. G. Taboada,${ }^{3}$ H. C. M. van Genuchten, ${ }^{1}$ M. Bozkurt, ${ }^{1}$ V. M. Fomin,,${ }^{1,45, *}$ \\ V. N. Gladilin, ${ }^{4,5}$ D. Granados, ${ }^{3}$ J. M. García, ${ }^{3}$ P. C. M. Christianen, ${ }^{2}$ J. C. Maan, ${ }^{2}$ J. T. Devreese,,${ }^{1,4}$ and P. M. Koenraad ${ }^{1}$ \\ ${ }^{1}$ PSN, COBRA, Eindhoven University of Technology, NL-5600 MB Eindhoven, The Netherlands \\ ${ }^{2}$ HFML, IMM, Radboud University Nijmegen, NL-6525 ED Nijmegen, The Netherlands \\ ${ }^{3}$ CSIC, Instituto de Microelectrónica de Madrid, E-28760 Tres Cantos, Spain \\ ${ }^{4}$ TFVS, Universiteit Antwerpen, B-2020 Antwerpen, Belgium \\ ${ }^{5}$ FSM, Universitatea de Stat din Moldova, MDV-2009 Chisinau, Moldova
}

(Received 6 April 2009; revised manuscript received 21 September 2009; published 16 October 2009)

\begin{abstract}
We investigate the exciton energy level structure of a large ensemble of InAs/GaAs quantum rings by photoluminescence spectroscopy in magnetic fields up to $30 \mathrm{~T}$ for different excitation densities. The confinement of an electron and a hole in these type I quantum rings along with the Coulomb interaction suppress the excitonic Aharonov-Bohm effect. We show that the exciton energy levels are nonequidistant and split up in only two levels in magnetic field, reflecting the ringlike geometry. A model, based on realistic parameters of the self-assembled quantum rings, allows us to interpret the essential features of the observed PL spectra in terms of the calculated optical transition probabilities.
\end{abstract}

DOI: $10.1103 /$ PhysRevB.80.155318

PACS number(s): 73.21.La, 78.67.Hc

The excitonic energy structure of self-assembled quantum dots (QDs) is well studied. ${ }^{1,2}$ By magnetoluminescence experiments it has been demonstrated that the electronic energy levels in a QD can be described by the Fock-Darwin model for a two-dimensional harmonic oscillator in a magnetic field. ${ }^{3-9}$ Changing the QDs to ringlike structures modifies the energy spectrum, and gives rise to the Aharonov-Bohm (AB) effect: the oscillatory behavior of charge carriers in a ringlike geometry as a function of the magnetic flux threading the opening of the ring. ${ }^{10}$ If the magnetic field penetrates into the conducting region of the ring, the AB-type oscillations due to the magnetic flux threading the opening coexist with the diamagnetic shift of energy levels and are aperiodic (see, e.g., Refs. 11 and 12).

The optical emission of self-assembled InAs/GaAs quantum rings (QRs) (Refs. 13 and 14) has been studied experimentally without a magnetic field, ${ }^{15}$ and in magnetic fields not higher than $9 \mathrm{~T}^{16}$ In general, excitons are neutral excitations, thus on forehand we do not expect any sensitivity to the magnetic flux. However, since the exciton is a polarizable composite particle, the area between the different trajectories of the electron and the hole determines the phase picked up by the exciton. ${ }^{17}$ Therefore the possible prominence of the $\mathrm{AB}$ effect for excitons strongly depends on their polarization. Calculations of the photoluminescence (PL) spectra of type I GaAs/AlGaAs and several type II QRs showed that a weak reminiscent feature of the $\mathrm{AB}$ effect in the PL spectrum might be observed. ${ }^{18,19}$ Experimentally the optical AB effect has been shown in different ringlike structures. ${ }^{20-22}$ Recently, the exciton energy spectra for various models of the InAs/ GaAs self-assembled QRs were calculated as a function of the applied magnetic field and it was shown that the spectra are very sensitive to the details of the QR shape. ${ }^{23}$

In this paper we consider the excitonic properties of selfassembled InAs/GaAs QRs in magnetic fields up to $30 \mathrm{~T}$. Using different excitation densities we probe the magneto-PL of the ground and excited states. The essential features in the magneto-PL spectra are reproduced in calculations based on a realistic QR model. ${ }^{23-25}$ We will demon- strate that QRs have nonequidistant energy levels and exhibit a magnetic field induced splitting of the higher excitonic energy levels into two levels, in contrast to the $n+1$ fold degeneracy of the $n$th excited state of QDs with a harmonic confinement potential. Furthermore, we will show that the confinement of an electron and a hole along with the Coulomb interaction suppress the excitonic $\mathrm{AB}$ effect in these QRs.

For the PL studies, a sample containing a single layer of QRs (Refs. 13 and 14) is mounted in a liquid-helium bath cryostat at $T=4.2 \mathrm{~K}$. The excitation is provided by a Dye laser operating at $2 \mathrm{eV}$. The excitation power is varied with a Babinet-Soleil compensator in combination with a linear polarizer. A Wollaston prism allows for simultaneous detection of both circular polarizations. The PL signal is dispersed by a single grating spectrometer, and the detection is performed by a liquid-nitrogen-cooled charge-coupled device camera. Static magnetic fields up to $30 \mathrm{~T}$ were applied parallel to the growth direction and the PL is detected in the Faraday configuration.

The dependence of the QR emission energy on the excitation density is shown in Fig. 1(a). The ground-state emission energy of the QRs is centered around $1.308 \mathrm{eV}$, typical for these nanostructures. ${ }^{14}$ The ground-state emission has an inhomogeneous broadening with a full width at half maximum of $20 \mathrm{meV}$. With increasing excitation density two additional peaks can be resolved. These peaks have an energy of 39 and $63 \mathrm{meV}$ above the ground-state energy. The wetting layer (WL) emission is centered around $1.438 \mathrm{eV}$ (not shown), which is $67 \mathrm{meV}$ above the highest observed confined-state energy of the QRs.

We determine the energy of the ground-state PL by fitting the spectra at low excitation densities by a Gaussian. The observed ground-state emission energy $E(B)$ of an exciton in a QR for relatively small $B$ is approximately given by $E(B)=E_{0} \pm \frac{1}{2} g_{\text {ex }} \mu_{B} B+\alpha_{d} B^{2}{ }^{25}$ Here $E_{0}$ is the emission energy at $B=0 \mathrm{~T}, g_{\text {ex }}$ is the exciton $g$ factor, $\mu_{B}=+5.79$ $\times 10^{-5} \mathrm{eV} / \mathrm{T}$ is the Bohr magneton, and $\alpha_{d}$ is the diamag- 

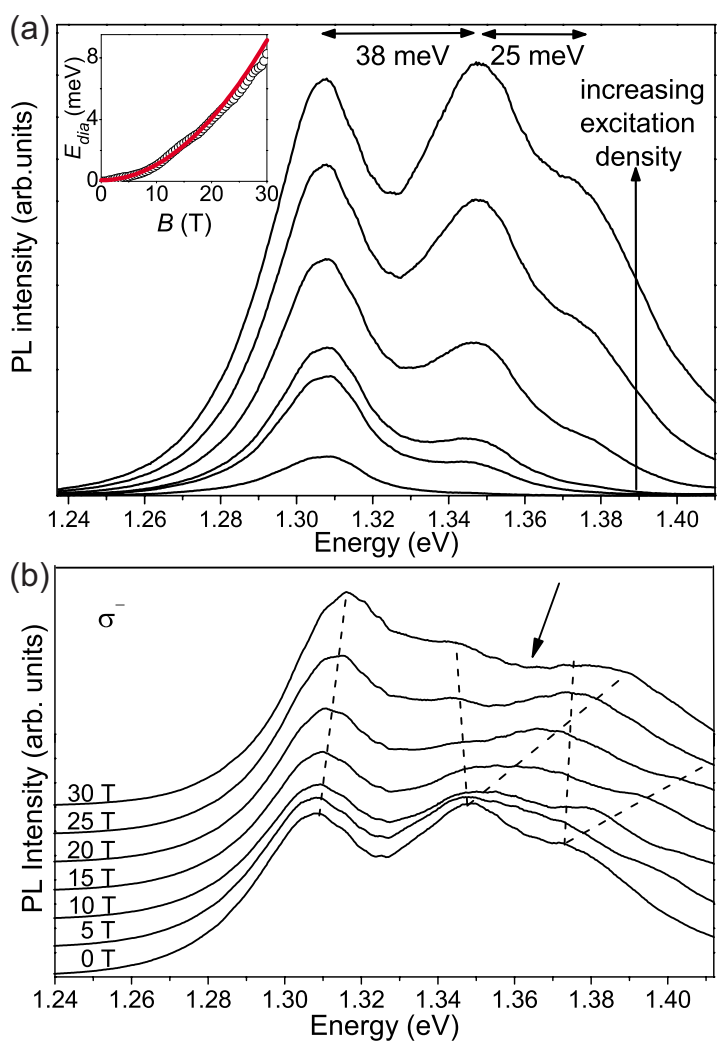

FIG. 1. (Color online) (a) PL as a function of excitation density, for which the lowest (highest) excitation density is $10^{2} \mathrm{~W} \mathrm{~cm}^{-2}$ $\left(10^{5} \mathrm{~W} \mathrm{~cm}^{-2}\right)$. Two excited states can be distinguished for higher excitation density located 38 and $63 \mathrm{meV}$ above the ground-state emission energy. The inset shows the diamagnetic shift $E_{\text {dia }}$ of the ground state. The quadratic fit (red line) is used to determine the diamagnetic coefficient $\alpha_{d}$. (b) Excited states as a function of $B$ in $\sigma^{-}$polarization for an excitation density of $10^{5} \mathrm{~W} \mathrm{~cm}^{-2}$. The dashed lines are guides to the eye in order to follow the evolution of the peak positions in $B$. The arrow indicates the emission energy at which for QDs a third peak is present. As opposed to QDs we observe a minimum in PL intensity.

netic coefficient. The second term is the Zeeman term which gives rise to a spin-induced splitting of the exciton PL in a magnetic field. We define $g_{e x}=\frac{E\left(\sigma^{+}\right)-E\left(\sigma^{-}\right)}{\mu_{B} B}$, and find $g_{e x}$ $=-1.7$, in correspondence with previously reported values obtained on individual QRs and comparable to values for QDs. ${ }^{20,26}$ In the inset of Fig. 1(a) the diamagnetic shift $E_{\text {dia }}$ is shown, defined by $E_{d i a}=\frac{E\left(\sigma^{-}\right)+E\left(\sigma^{+}\right)}{2}-E_{0}$. The diamagnetic shift has a smooth dependence on the magnetic field. From the quadratic fit (solid line) we find $\alpha_{d}=10 \mu \mathrm{eV} / \mathrm{T}^{2}$, in agreement with previous reported values for QRs (Ref. 16) and QDs. ${ }^{26}$

To investigate the influence of the ringlike geometry on the excitonic behavior in the excited states of the QRs, we measured the magneto-PL of these structures for higher excitation intensities. Figure 1(b) shows the higher excitation data in $\sigma^{-}$polarization as function of $B$ in intervals of $5 \mathrm{~T}$. The dashed lines are a guide to the eye and follow the peak positions. We have carefully assigned the PL peak positions as function of $B$ by comparing the PL spectra at different $B$ (see Fig. 2). As implied by Fig. 1(b), both resolvable excited

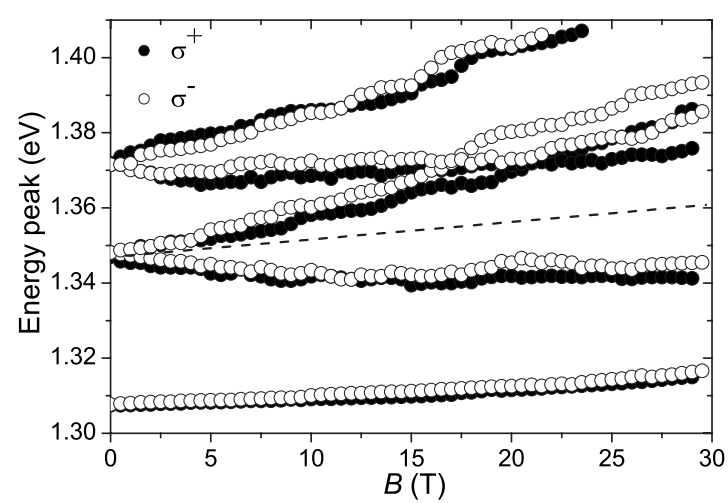

FIG. 2. The energy diagram showing the peak position in $B$ in both $\sigma^{-}$(empty circles) and $\sigma^{+}$(filled circles) polarization. The QRs exhibit splittings into two states of the different excited states, in contrast to QDs where a third peak (indicated by the dashed line) is observed.

states split up in two separate peaks. Each of the PL peaks of the QRs Zeeman splits further with a smaller energy separation into two peaks of opposite circular polarization.

To understand the energy structure of the excitons we use a model based on the structural properties of these QRs obtained by cross-sectional scanning tunneling microscopy measurements. ${ }^{12,24,27}$ The model is used to calculate the single-exciton optical transition probability spectrum. ${ }^{23}$ The results are shown in Figs. 3(a) and 3(b), for a noninteracting and an interacting electron-hole pair, respectively. For a noninteracting electron-hole pair we calculate that around $B$ $=15 \mathrm{~T}$ there is a crossover in the ground-state energy, in agreement with magnetization experiments. ${ }^{28}$ The inclusion of the Coulomb interaction results into a smooth behavior of the ground-state energy as function of $B$, as shown in Fig. 3(b). At a magnetic field of $15 \mathrm{~T}$ the calculated spectrum shows that the first-excited state has a reduced optical spectral probability. This is due to the redistribution of the oscillator strength between the first-excited state and the ground state in favor of the latter. In the case of the interacting electron-hole pair the ground-state energy is lowered by the energy of the electron-hole Coulomb attraction, which is 13 meV.

In the following we compare the experimental results with the theoretical calculations. The calculated ground-state emission energy is $1.34 \mathrm{eV}$, in reasonable agreement with the measured ground-state PL energy $(1.31 \mathrm{eV})$. In general we find that the calculated energies are $\sim 30 \mathrm{meV}$ higher than the experimental values. The PL of the continuum states in the $\mathrm{WL}$ is calculated to be at $1.43 \mathrm{eV}$, which corresponds well to the measured value of $1.44 \mathrm{eV}$. Moreover, the calculations show at $B=30 \mathrm{~T}$ a $10 \mathrm{meV}$ shift of the ground state to higher energy, where the experimental value is $8 \mathrm{meV}$. The Zeeman effect is not taken into account in the calculations. Both experimentally and theoretically we find a smooth dependence of the ground-state emission energy on $B$, which is a consequence of the Coulomb interaction.

To interpret the higher lying energy states, we will focus only on the states in the model having a large spectral transition probability [cf. Fig. 3(b)], and compare them with the experimentally observed PL peaks. The first-excited state is 

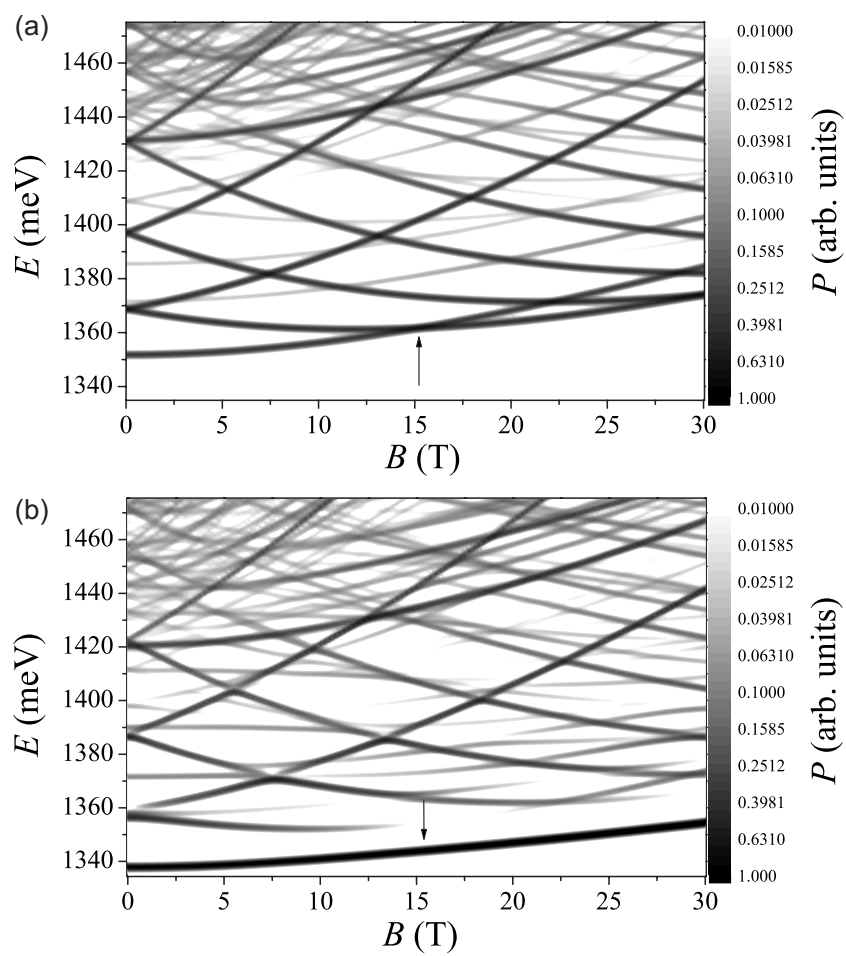

FIG. 3. Calculated optical transition probabilities for a realistic QR in the case of (a) a noninteracting electron-hole pair and (b) an interacting electron-hole pair. The gray scale is logarithmic where black (white) corresponds to the highest (lowest) transition probability. The arrows correspond to the first excitonic $\mathrm{AB}$ resonance in the ground state.

expected at $20 \mathrm{meV}$ above the ground-state emission energy. However, in our experimental data we cannot resolve this peak due to the inhomogeneous broadening. The secondexcited state in our model is at $58 \mathrm{meV}$ above the groundstate emission energy and corresponds to the second peak in our experiment, whereas the calculated energy level at 1.42 $\mathrm{eV}, 82 \mathrm{meV}$ above the ground-state emission, corresponds to the third peak we observe. In order to better compare the calculated spectra to the experimental spectra we introduce a Gaussian broadening $\Gamma$, which simulates the inhomogeneous broadening of the ensemble. For $\Gamma=10 \mathrm{meV}$, we find the best comparison of the calculated spectra with the experimental data. Figure 4 shows the calculated PL spectra for $B$ up to $30 \mathrm{~T}$ in steps of $5 \mathrm{~T}$. The calculated and measured spectra [cf. Fig. 1(b)] show a qualitative resemblance, although the absolute values of the energy splittings are different. Importantly, the introduced broadening indeed shows that the first-excited state is not resolvable in the magnetoPL. We do note that based on our model we assign the measured PL peaks to different excitonic states in the QRs as compared to the identification based on PLE measurements on single QRs. ${ }^{29}$ However, within the theoretical model, which was successfully applied to explain the magnetization behavior of QRs on similar samples, ${ }^{12,28}$ we found that for all realistic ring parameters the PL of the first-excited state is concealed by the ground-state luminescence if an inhomogeneous broadening of about $20 \mathrm{meV}$ is included.

The excitonic behavior characteristic for ringlike struc-

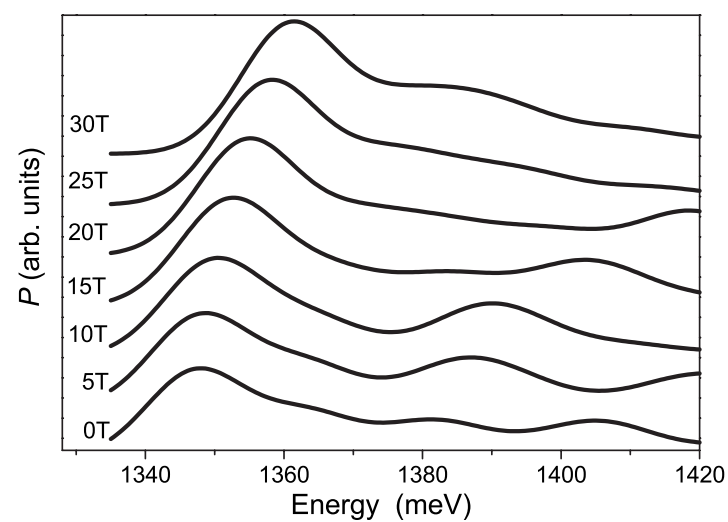

FIG. 4. Calculated broadened optical transition probabilities $P$ as a function of the emission energy $E$ for $B=0$ to $30 \mathrm{~T}$ in $5 \mathrm{~T}$ steps.

tures manifests itself in the magneto-PL under high excitation conditions. We observe the splitting of the excited states into two states as well as nonequidistant energy level splittings. In contrast to our measurements, experiments on QDs resulted in a magnetic induced splitting of the $d$ state into three states and equidistant energy levels. ${ }^{5,6,8}$ This $d$ state corresponds to the second peak in Fig. 1(b), which for QRs has predominantly an $l=2$ character, where $l$ is the orbital angular momentum quantum number. The dashed line in Fig. 2 indicates the position of the third energy level as observed for QDs. However, we observe a minimum in PL intensity at this emission energy [see arrow in Fig. 1(b)], indicating the absence of this third peak.

In the calculations the strongest effect on the oscillator strength is expected for the first-excited state with predominantly an $l=1$ character. This state is not resolved in our measurements due to the inhomogeneous broadening. The oscillator strength of the ground state of the single exciton of our modeled QR does not significantly change with $B$, as was confirmed in the experiments. In contrast to the AB effect of single electrons in these rings, we do not observe nor expect an excitonic AB effect based on our model. The absence of prominent oscillations in the ground-state energy of the calculated exciton spectra as compared to the case of a noninteracting electron-hole pair is a consequence of the Coulomb interaction. A charge-tunable QR sample will allow for the control of the charged state of the excitons in the $\mathrm{QR},{ }^{15}$ giving us the ability to study better the influence of the Coulomb interaction on the $\mathrm{AB}$ effect in these QRs.

The details of the calculated spectra are very sensitive to the size, shape, and composition of the QR and it is difficult to find a quantitative agreement between the calculated optical transition probabilities and the measured PL spectra. ${ }^{23}$ The model used does not include many-exciton complexes and charged excitonic states, which influence the optical transition probability spectra. We estimate this will only be a small effect as the exciton binding energy is an order of magnitude larger compared to the exciton-exciton interaction and additional charging energies. In order to calculate the actual PL spectra from the optical transition probability spectra, one needs to take in account a nonequilibrium distribution function for excitons in a strong laser field and use a response theory. ${ }^{30,31}$ Despite the preliminary character of our 
model, we are able to find a qualitative agreement between the measurements and the calculations and thereby we can explain the essential features in our measurements.

To conclude, we have analyzed the emission energy of a large ensemble of self-assembled InAs/GaAs QRs in high magnetic fields. Our model shows that the confinement of an electron and a hole along with the Coulomb interaction suppress the excitonic $\mathrm{AB}$ effect in these nanostructures. The ring character of our nanostructures results in nonequidistant energy level splittings in the exciton diagram and into a magnetic field induced splitting of each excited state into two states. This is in contrast to what has been observed in QD measurements. The optical transition probabilities are calculated within our model, based on the characterization of a realistic QR. Comparing these calculations with our experimental data we find a qualitative agreement, which allows us to identify the different PL peaks and helps to explain the excitonic behavior in magnetic field.

This work is part of the research program of NanoNed and FOM, which are financially supported by the NWO (The Netherlands). This work was supported by the FWO-V (Projects No. G.0435.03 and No. G.0449.04), the WOG (Grant No. WO.035.04N, Belgium), the NANINPHO-QD, MICIN (Grant No. TEC2008-06756-C03-01), the MEC (Consolider-Ingenio 2010 "QOIT," Grant No. CSD200600019, Spain), and the EC SANDiE Network of Excellence (Grant No. NMP4-CT-2004-500101).
*Present address: Institute for Integrative Nanosciences, IFW Dresden, D-01069 Dresden, Germany.

${ }^{1}$ M. Bayer, O. Stern, P. Hawrylak, S. Fafard, and A. Forchel, Nature (London) 405, 923 (2000).

${ }^{2}$ R. J. Warburton, B. T. Miller, C. S. Dürr, C. Bödefeld, K. Karrai, J. P. Kotthaus, G. Medeiros-Ribeiro, P. M. Petroff, and S. Huant, Phys. Rev. B 58, 16221 (1998).

${ }^{3}$ V. Fock, Z. Phys. 47, 446 (1928).

${ }^{4}$ C. Darwin, Proc. Cambridge Philos. Soc. 27, 86 (1931).

${ }^{5}$ A. Babinski, M. Potemski, S. Raymond, J. Lapointe, and Z. R. Wasilewski, Phys. Rev. B 74, 155301 (2006).

${ }^{6}$ S. Awirothananon, S. Raymond, S. Studenikin, M. Vachon, W. Render, A. Sachrajda, X. Wu, A. Babinski, M. Potemski, S. Fafard, S. J. Cheng, M. Korkusinski, and P. Hawrylak, Phys. Rev. B 78, 235313 (2008).

${ }^{7}$ R. K. Hayden, K. Uchida, N. Miura, A. Polimeni, S. T. Stoddart, M. Henini, L. Eaves, and P. C. Main, Physica B 249-251, 262 (1998).

${ }^{8}$ S. Raymond, S. Studenikin, A. Sachrajda, Z. Wasilewski, S. J. Cheng, W. Sheng, P. Hawrylak, A. Babinski, M. Potemski, G. Ortner, and M. Bayer, Phys. Rev. Lett. 92, 187402 (2004).

${ }^{9}$ J. H. Blokland, F. J. P. Wijnen, P. C. M. Christianen, U. Zeitler, J. C. Maan, P. Kailuweit, D. Reuter, and A. D. Wieck, Phys. Rev. B 75, 233305 (2007).

${ }^{10}$ Y. Aharonov and D. Bohm, Phys. Rev. 115, 485 (1959).

${ }^{11}$ W. C. Tan and J. C. Inkson, Phys. Rev. B 60, 5626 (1999).

${ }^{12}$ V. M. Fomin, V. N. Gladilin, S. N. Klimin, J. T. Devreese, N. A. J. M. Kleemans, and P. M. Koenraad, Phys. Rev. B 76, 235320 (2007).

${ }^{13}$ J. M. García, G. Medeiros-Ribeiro, K. Schmidt, T. Ngo, J. L. Feng, A. Lorke, J. Kotthaus, and P. M. Petroff, Appl. Phys. Lett. 71, 2014 (1997).

${ }^{14}$ D. Granados and J. M. García, Appl. Phys. Lett. 82, 2401 (2003).

${ }^{15}$ R. J. Warburton, C. Schäflein, D. Haft, F. Bickel, A. Lorke, K. Karrai, J. M. Garcia, W. Schoenfeld, and P. M. Petroff, Nature (London) 405, 926 (2000).
${ }^{16}$ D. Haft, C. Schulhauser, A. O. Govorov, R. J. Warburton, K. Karrai, J. M. Garcia, W. Schoenfeld, and P. M. Petroff, Physica E 13, 165 (2002).

${ }^{17}$ A. O. Govorov, S. E. Ulloa, K. Karrai, and R. J. Warburton, Phys. Rev. B 66, 081309(R) (2002).

${ }^{18}$ M. Grochol, F. Grosse, and R. Zimmermann, Phys. Rev. B 74, 115416 (2006).

${ }^{19}$ J. I. Climente, J. Planelles, and W. Jaskólski, Phys. Rev. B 68, 075307 (2003).

${ }^{20}$ M. Bayer, M. Korkusinski, P. Hawrylak, T. Gutbrod, M. Michel, and A. Forchel, Phys. Rev. Lett. 90, 186801 (2003).

${ }^{21}$ I. R. Sellers, V. R. Whiteside, I. L. Kuskovsky, A. O. Govorov, and B. D. McCombe, Phys. Rev. Lett. 100, 136405 (2008).

${ }^{22}$ E. Ribeiro, A. O. Govorov, W. Carvalho, Jr., and G. MedeirosRibeiro, Phys. Rev. Lett. 92, 126402 (2004).

${ }^{23}$ V. M. Fomin, V. N. Gladilin, J. T. Devreese, N. A. J. M. Kleemans, M. Bozkurt, and P. M. Koenraad, Phys. Status Solidi B 245, 2657 (2008).

${ }^{24}$ V. M. Fomin, V. N. Gladilin, J. T. Devreese, N. A. J. M. Kleemans, and P. M. Koenraad, Phys. Rev. B 77, 205326 (2008).

${ }^{25}$ S. N. Walck and T. L. Reinecke, Phys. Rev. B 57, 9088 (1998).

${ }^{26}$ C. Schulhauser, D. Haft, R. J. Warburton, K. Karrai, A. O. Govorov, A. V. Kalameitsev, A. Chaplik, W. Schoenfeld, J. M. Garcia, and P. M. Petroff, Phys. Rev. B 66, 193303 (2002).

${ }^{27}$ P. Offermans, P. M. Koenraad, J. H. Wolter, D. Granados, J. M. García, V. M. Fomin, V. N. Gladilin, and J. T. Devreese, Appl. Phys. Lett. 87, 131902 (2005).

${ }^{28}$ N. A. J. M. Kleemans, I. M. A. Bominaar-Silkens, V. M. Fomin, V. N. Gladilin, D. Granados, A. G. Taboada, J. M. García, P. Offermans, U. Zeitler, P. C. M. Christianen, J. C. Maan, J. T. Devreese, and P. M. Koenraad, Phys. Rev. Lett. 99, 146808 (2007).

${ }^{29}$ B. Alén, J. Martínez-Pastor, D. Granados, and J. M. García, Phys. Rev. B 72, 155331 (2005).

${ }^{30}$ R. Kubo, J. Phys. Soc. Jpn. 12, 570 (1957).

${ }^{31}$ V. M. Fomin, V. N. Gladilin, J. T. Devreese, E. P. Pokatilov, S. N. Balaban, and S. N. Klimin, Phys. Rev. B 57, 2415 (1998). 